\title{
TAIGA-IACT poining control and monitoring software status
}

D. P. Zhurov, ${ }^{a, b, *}$ O. A. Gress ${ }^{a}$ and D. S. Lukyantsev ${ }^{a}$ on behalf of the TAIGA Collaboration (a complete list of authors can be found at the end of the proceedings)

${ }^{a}$ Applied Physics Institute of Irkutsk State University, API ISU, Irkutsk, Russia

${ }^{b}$ Irkutsk National Research Technical University, INRTU, Irkutsk, Russia

E-mail: sidney28@yandex.ru

The TAIGA-IACTs are part of the hybrid TAIGA experimental complex, located near lake Baikal in Tunka valley, Siberia, Russia. The telescopes have segmented mirrors in Davis-Cotton design with the reflector diameter of $4.3 \mathrm{~m}$ and an imaging camera with PMTs in its focus to detect nanosecond flashes of Cherenkov light from EAS. The TAIGA-IACTs are operating in wobble mode. Their operation requires high pointing and tracking accuracy, especially important for long exposure times. The telescope positioning system consists of steppers motors, 17-bit angular encoders and a CCD camera for accurate monitoring of the telescope pointing by stars in its field of view and related calibration procedures. The telescope is controlled by using the custom software based on the EPICS (Experimental Physics and Industrial Control System) package. This report presents an overview of the TAIGA-IACT control and monitoring software, pointing accuracy and the relevant calibration procedures.

\footnotetext{
*** 37th International Cosmic Ray Conference (ICRC2021), ***

*** 12-23 July $2021 * * *$

*** Berlin, Germany - Online ***
}

\footnotetext{
${ }^{*}$ Presenter
} 


\section{Introduction}

The TAIGA observatory $[1,2]$ is designed for high-energy gamma-ray astronomy and cosmicray physics with a new, hybrid detection concept[3]. This hybrid approach combines the detection of the Cherenkov light emitted by extensive air showers (EAS) with two different detector systems: with the timing array TAIGA-HiSCORE and with imaging air Cherenkov telescopes TAIGAIACT. Since 2020 two IACT telescopes are installed and operate on the TAIGA site. The third telescope is in deployment and will be commissioned and put in operation in 2021. The results of analysis of the TAIGA-IACT data can be found in $[4,5]$. In order to control the telescope hardware devices, dedicated software was developed. The software was developed using EPICS ${ }^{1}$ framework. Required telescope pointing accuracy is achieved by developed telescope pointing model and calibration approach. The software and the approach are described in this work.

\section{The TAIGA-IACT telescopes}

The TAIGA-IACT telescope has an alt-azimuth mount and a camera in the focus of a segmented Davis-Cotton design reflector. The telescope reflector is $4.3 \mathrm{~m}$ in diameter and is composed of 34 mirror segments. The focal length of the telescope is $4.75 \mathrm{~m}$. The camera has $9.6^{\circ}$ field of view and $0.36^{\circ}$ pixel resolution. It consists of 560 (the 1st telescope) and 595 (the 2nd and 3rd telescope) PMTs equipped with Winston cones.

For the pointing calibration and measurements, a CCD camera Prosilica GT1380 is installed on the dish near the mirrors. The field of view of the CCD camera is wider than the telescope camera and is equal to $31.4^{\circ} \times 23.6^{\circ}\left(0.023^{\circ}\right.$ per pixel). The CCD camera captures on the same image the sky region with the field of view of the telescope camera and the telescope camera with eight positioning LEDs installed along the perimeter. The LEDs are dedicated to measuring the telescope camera position on the CCD-camera images. As the telescope camera does not allow to perform astrometry by stars directly, a special white calibration screen was developed. The calibration screen is equipped with a motor and gears and can be rolled and unrolled remotely. During a calibration run, a bright star is focused on the unrolled screen and its focused image can be seen on the screen using CCD camera. The drive system of the telescope is consists of Phytron hybrid stepper motors, 17-bit shaft encoders, and limit switches, all are connected to the PhyMOTION control unit.

\subsection{The TAIGA-IACT telescope control software}

To provide unified access to the telescope hardware control and readout and simplify software development EPICS framework is used. A diagram of telescope hardware and software is presented in figure 1. The motor, CCD camera, and temperature readout are controlled by the appropriate driver in the telescope control IOC. The on-the-fly CCD-image processing for telescope direction estimation is implemented using the AreaDetector plugin for the EPICS system that is included in the telescope control IOC. The FITS file format is used for CCD-camera image storage. Astrometry.net[6] ${ }^{2}$ software is used for processing of the telescope field of view region on CCD-camera images.

\footnotetext{
${ }^{1}$ https://epics.anl.gov

${ }^{2} \mathrm{https} / / /$ github.com/dstndstn/astrometry.net
} 


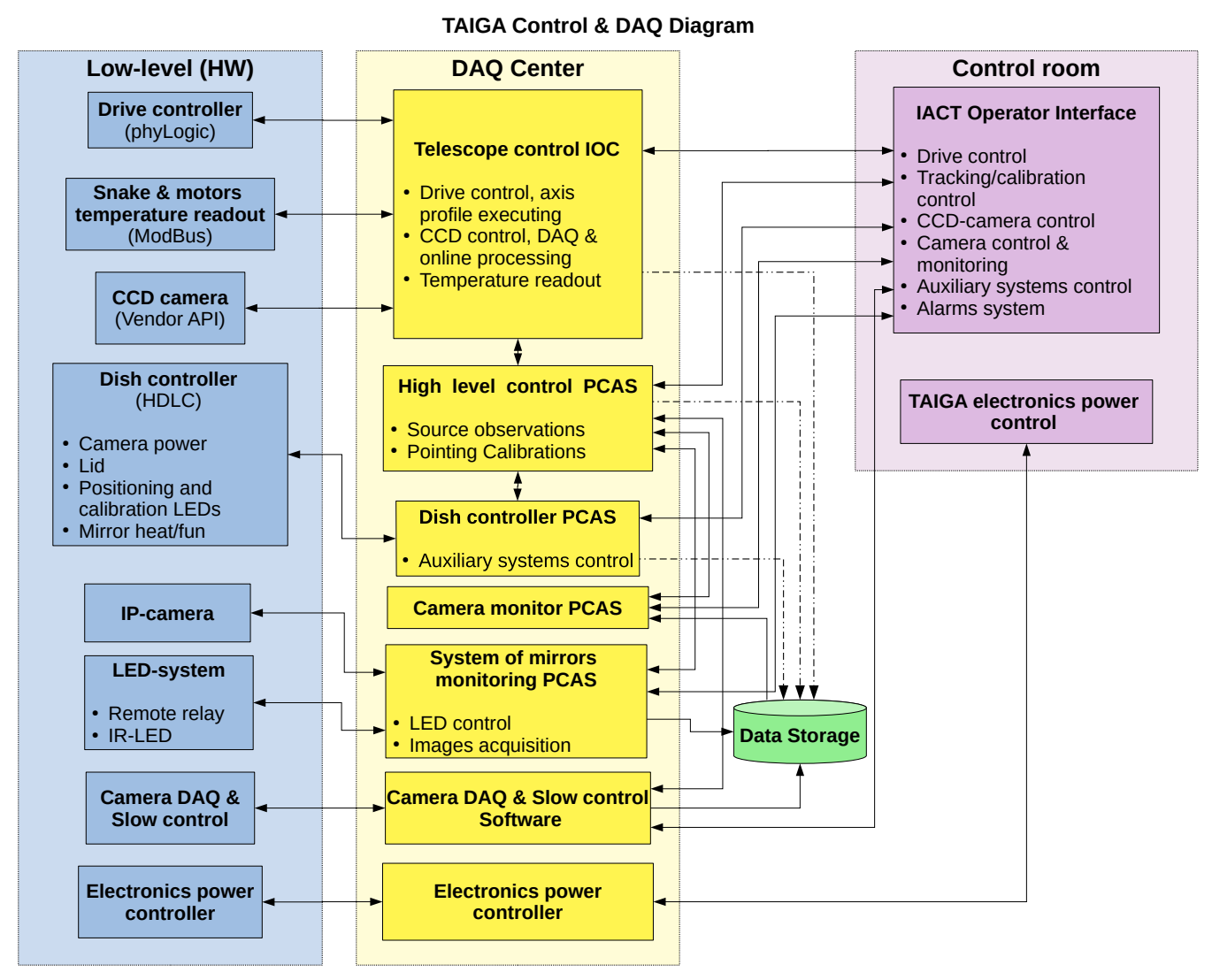

Figure 1: The TAIGA-IACT control and data acquisition software diagram.

Two PCAS (Portable Channel Access Server) are written on Python for auxiliary systems control and high-level automation of the telescope tracking and calibration operations. The highlevel control PCAS configure telescope runs and performs trajectory calculation, hardware setting, and control of the telescope operation execution. The telescope trajectory is calculated using the SOFA $^{3}$ software and applying the telescope pointing model to minimize telescope pointing errors. The on-the-fly CCD image processing allows telescope direction estimation and takes its results into account to correct the telescope trajectory online. The IP-camera and the IR-LED installed on the telescope camera are dedicated to monitor telescope mirrors status (frost detection).

The operator GUI is developed using the EPICS Qt framework and provides hardware control and monitoring of the telescope status and parameters. The operator GUI provides a graphical representation of parameters, color indication, and audio alarm system.

\section{The TAIGA-IACT telescope pointing calibration}

For the telescope pointing a transformation chain presented in figure 1 is used. The telescope direction can be estimated by the telescope shaft encoders and by the CCD camera installed on the dish. Both methods are based on models which parameters are determined experimentally by special measurements and tracking known bright stars.

${ }^{3}$ http://www.iausofa.org/ 


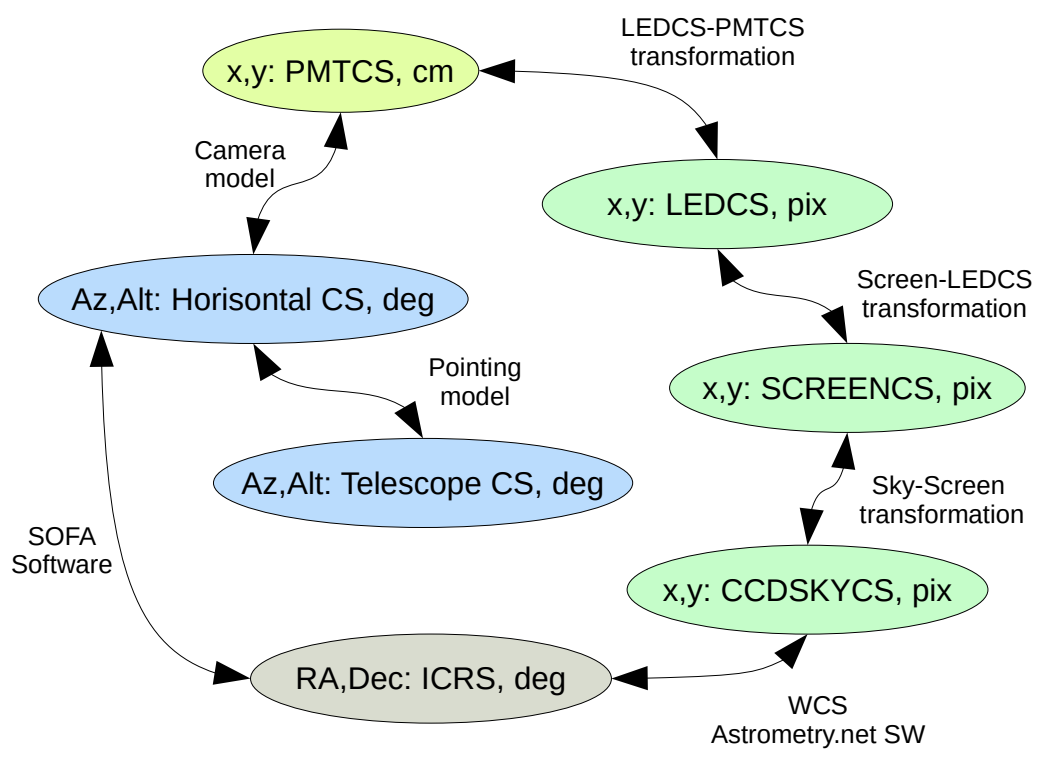

Figure 2: Telescope coordinates transformation graph. To estimate the telescope direction, encoders and the CCD camera is used. To transform encoder readouts to the telescope direction in the horizontal coordinate system a pointing model is used. The direction of the telescope in the ICRS (International Celestial Reference System) coordinate system can be estimated by CCD camera images. The WCS transformation parameters are determined using Astrometry.net software. Transformations from the ICRS to the horizontal coordinate system are performed using the SOFA library.

The first step of the telescope calibration is measurements of reference positions of the positioning LEDs and determination of the transformation to the PMT coordinate system. Measurement starts in twilight when the hexagonal outlines of Winston cones of the telescope camera can be seen on CCD camera images. The dependency of positions of the LEDs on different telescope positions by altitude angle is also determined.

A simple camera model[7] is used to predict the position of a source projection on the focal plane and determine the position of the telescope during the pointing calibration runs with the calibration screen.

\subsection{The pointing model}

The telescope direction in the horizontal coordinate system can be estimated by the shaft encoders using the telescope pointing model. The model is designed to predict and correct telescope pointing deviations caused by different reasons. The most prominent reasons for such deviations are offsets of the shaft encoders, small telescope tilts, bending of the telescope mount, non-perpendicular alignment of the altitude axis relative to the telescope optical axis, non-perpendicular alignment of the altitude axis relative to the azimuth axis. We use a slightly modified pointing model that has previously been successfully used in other telescopes [8,9]. Speaking of another new approach for the telescope pointing modeling (not used in this work) you can see more in [10].

For the pointing model measurements, the calibration screen inside the telescope camera is unrolled and during a night the telescope tracks several bright stars consistently, automatic switching between targets. Using the spot analysis algorithm the position of the star image on the screen is 
determined and then transformed to the focal plane coordinate system. The telescope direction in the horizontal coordinate system is estimated by the telescope camera model.

\subsection{Transformation from the CCD to telescope camera}

To determine the telescope position by the CCD camera a transformation of the telescope camera center to the CCD sky region is determined. It takes into account the possibility of small displacements of the CCD camera direction concerning the telescope optical axis and the possible dependence on the telescope altitude[11]. The parameters of the transformation are determined experimentally using bright stars and the calibration screen unrolled in the telescope camera.

The transformation is used for the telescope direction estimation and transformation in the vicinity of the camera center. The telescope camera model is used to calculate the position of an arbitrary star on the telescope's focal plane.

\section{The TAIGA-IACT pointing results}

The telescope pointing calibration measurements with the unrolled screen are regularly performed between the observation periods in a small moon in order not to waste the observation time. The pointing model estimation of the telescope direction has smooth not static systematic. An experiment with unrolled calibration screen showed that the direction estimation using the CCD camera does not have such systematic. The telescope trajectory is reconstructed with the CCD camera corrections to take it into account. The corrections are performed online during the tracking and offline for future data analysis

\subsection{The spot position prediction and telescope direction estimation by CCD camera}

By the data with unrolled screen difference between the prediction of the source position by the telescope camera model using direction estimated using the CCD camera and measured spot position has about 0.01 degrees standard deviation by both axes. The mean offset of measured positions from predicted positions of a bright star image does not exceed 0.01 degrees by both axes for calibration data during the 2020-2021 season. The calibration gives good accuracy for the entire season and it has good time stability.

\subsection{Pointing measurements using anode current on telescope camera PMTs}

As an additional independent test of the CCD-camera transformation accuracy, special runs are performed. The method based on the measurements of anode current on the PMTs during a crosslike scan of a bright star[12]. The procedure for such measurements is the following: telescope track a bright star to put its spot in the target telescope camera pixel. During the measurements, the calibration screen is rolled, HV on the camera PMTs is on, the currents and the axis position by encoder readouts have $1 \mathrm{~Hz}$ frequency. The telescope moves in such a way, to change the position of the spot by $\mathrm{x}$ and $\mathrm{y}$ coordinate as presented in figure 3 (right). The telescope scans the profiles moving up, down, from left to right, and from right to left. Reference CCD camera images are obtained to apply the corrections.

For each passage, the dependence of anode current on $\mathrm{x}$ and $\mathrm{y}$ axes on the predicted bright star position is calculated. The full width at half maximum (FWHM) is calculated for the profiles. 
The deviation of the FWHM segment center from the PMT center position can be interpreted as an error of the star projection to the camera focal plane caused by the telescope direction estimation error or an error in the telescope camera model.

The results of such measurements for different altitude, different distances and directions from the camera center is presented on the figure 3 (left). The presented data show that the estimation of the source position on the telescope focal plane is not worse than 1 CCD camera pixel $(0.023$ degrees).
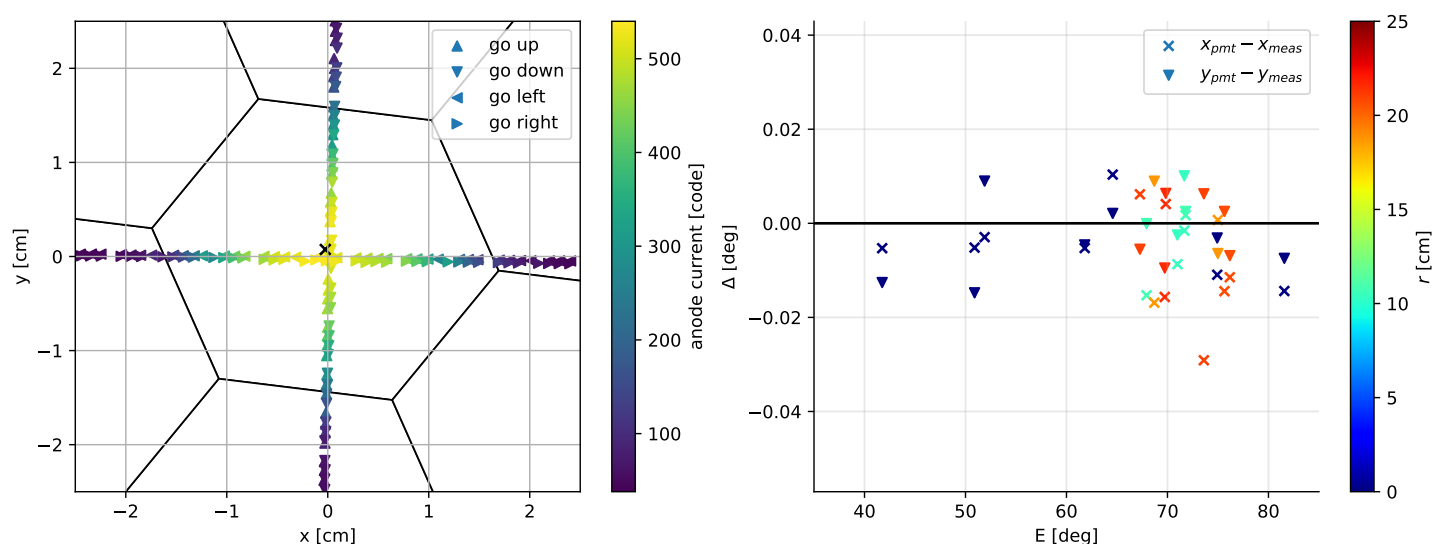

Figure 3: Left: an example of a trajectory of the start projection on the camera focal plane during the cross-like currents scan method. Right: The difference with the position of the target pixel on the telescope focal plane with the anode current profile FWHM segment center for the 2nd TAIGA-IACT telescope. The colors indicate the distance of the target pixel from the camera center. The abscissa shows the altitude of the telescope.

\section{Conclusions}

The TAIGA-IACT telescope control software is developed using EPICS framework. The telescope operates in wobble mode switching the direction every 20 minutes. The distance of the observed source from the telescope optical axis during the wobble mode is 1.2 degrees.

The telescope direction estimation using the pointing model is corrected by the CCD camera to achieve better accuracy. The telescope direction estimation by CCD camera takes into account possible small displacements of the CCD camera direction with respect to the telescope optical axis and the possible dependence on the telescope altitude. The CCD camera transformation calibration is time stable and the accuracy for the 2020-2021 season is better than 1 CCD pixel or 0.023 degrees. The position of a source on the telescope focal plane is calculated using the telescope camera model. The telescope pointing and a source position calculation accuracy were checked by the anode current, the result accuracy is not worse than 1 CCD camera pixel ( 0.023 degrees).

\section{Acknowledgments}

The work was performed at the UNU "Astrophysical Complex of MSU-ISU» (agreement 13.UNU.21.0007). The work is supported by Russian Foundation for Basic Research (grants N 
19-52-44002, 19-32-60003) the Russian Science Foundation (grant 19-72-20067 (Section 3), the Russian Federation Ministry of Science and High Education (projects FZZE-2020-0017, FZZE2020-0024) and Irkutsk State University (project N 091-20-308 and project N 091-21-305).

\section{References}

[1] Budnev, N., et al. "TAIGA — an advanced hybrid detector complex for astroparticle physics and high energy gamma-ray astronomy in the Tunka valley." Journal of Instrumentation 15.09 (2020): C09031.

[2] Budnev, N., et al. "The TAIGA - an advanced hybrid detector complex for astroparticle physics, cosmic ray physics and gamma-ray astronomy." PoS ICRC2021, 731 https://pos.sissa.it/395/731

[3] Kuzmichev, L., et al. "Experimental Complex TAIGA." Physics of Atomic Nuclei 83.9 (2020): 1375-1382.

[4] Sveshnikova, L., et al. "The detection of high energy gamma-rays ( larger $40 \mathrm{TeV}$ ) from Crab Nebular by a hybrid method of TAIGA installation" PoS ICRC2021, 861 https://pos.sissa.it/395/861

[5] Poreli, A., et al. "TAIGA-Observatory: First 5 years of operation of the HiSCORE AirCerenkov Array" PoS ICRC2021, 877 https://pos.sissa.it/395/877

[6] Lang, Dustin, et al. "Astrometry. net: Blind astrometric calibration of arbitrary astronomical images." The astronomical journal 139.5 (2010): 1782.

[7] Forsyth, David A., and Jean Ponce. "Computer Vision, A Modern Approach. Printice Hall." Upper Saddle River (2003).

[8] Lew, Bartosz. "Improving pointing of Torun 32-m radio telescope: effects of rail surface irregularities." Experimental Astronomy 45.1 (2018): 81-105.

[9] Sun, Z., et al. "The Establishment of Pointing Model for the Shanghai VGOS Radio Telescope." Journal of Physics: Conference Series. Vol. 1288. No. 1. IOP Publishing, 2019.

[10] Spengler, G., et al. "CTbend: A Baysian open-source framework to model pointing corrections of Cherenkov telescopes" PoS ICRC2021, 699 https://pos.sissa.it/395/699

[11] Zhurov, D., et al. "First results of the tracking system calibration of the TAIGA-IACT telescope." Journal of Physics: Conference Series. Vol. 1181. No. 1. IOP Publishing, 2019.

[12] Budnev, N., et al., "TAIGA - a hybrid array for high-energy gamma astronomy and cosmic-ray physics", Nuc. Instr. and Meth. in Phys. Res. Sec. A: Acc., Spectr., Det. and Ass. Equip., ISSN 0168-9002, 2019, https://doi.org/10.1016/j.nima.2019.04.067 


\section{Full Authors List: TAIGA Collaboration}

I. I. Astapov ${ }^{2}$ A. K. Awad ${ }^{10}$ P. A. Bezyazeekov ${ }^{3}$ M. Blank ${ }^{10}$ E. A. Bonvech ${ }^{1}$ A. N. Borodin ${ }^{4}$ A. V. Bulan ${ }^{1}$ M. Brueckner ${ }^{5}$ N. M. Budnev $^{3}$ A. Chiavassa ${ }^{6}$ D. V. Chernov ${ }^{1}$ A. N. Dyachok ${ }^{3}$ A. R. Gafarov ${ }^{3}$ A. Yu. Garmash ${ }^{7,8}$ V. M. Grebenyuk ${ }^{4,9}$ O. A. Gress ${ }^{3}$ E. Gress $^{3}$ T. I. Gress ${ }^{3}$ O. G. Grishin ${ }^{3}$ A. A. Grinyuk ${ }^{4}$ D. Horns ${ }^{10}$ N. N. Kalmykov ${ }^{1}$ V. V. Kindin ${ }^{2}$ S. N. Kiryuhin ${ }^{3}$ R. P. Kokoulin ${ }^{2}$ K. G. Kompaniets $^{2}$ E. E. Korosteleva ${ }^{1}$ V. A. Kozhin ${ }^{1}$ E. A. Kravchenko ${ }^{7,8}$ A. P. Kryukov ${ }^{1}$ L. A. Kuzmichev ${ }^{1}$ A. A. Lagutin ${ }^{11}$ M. Lavrova ${ }^{4}$ B. K. Lubsandorzhiev ${ }^{12}$ N. B. Lubsandorzhiev ${ }^{1}$ A. D. Lukanov ${ }^{12}$ D. S. Lukyantsev ${ }^{3}$ R. R. Mirgazov ${ }^{3}$ R. Mirzoyan ${ }^{13,3}$ R. D. Monkhoev ${ }^{3}$ E. A. Osipova ${ }^{1}$ A. L. Pakhorukov ${ }^{3}$ A. Pan $^{4}$ L. V. Pankov ${ }^{3}$ A. D. Panov ${ }^{1}$ A. A. Petrukhin ${ }^{2}$ D. A. Podgrudkov ${ }^{1}$ V. A. Poleschuk ${ }^{3}$ M. Popesku $^{14}$ E. G. Popova ${ }^{1}$ A. Porelli ${ }^{5}$ E. B. Postnikov ${ }^{1}$ V. V. Prosin ${ }^{1}$ V. S. Ptuskin ${ }^{15}$ A. A. Pushnin ${ }^{3}$ R. I. Raikin ${ }^{11}$ A. Y. Razumov ${ }^{1}$ G. I. Rubtsov $^{12}$ E. V. Ryabov ${ }^{3}$ Y. I. Sagan ${ }^{4,9}$ V. S. Samoliga ${ }^{3}$ A. A. Silaev ${ }^{1}$ A. A. Silaev(junior) ${ }^{1}$ A. Yu. Sidorenkov ${ }^{12}$ A. V. Skurikhin ${ }^{1}$ M. Slunecka $^{4}$ A. V. Sokolov ${ }^{7,8}$ L. G. Sveshnikova ${ }^{1}$ V. A. Tabolenko ${ }^{3}$ B. A. Tarashansky ${ }^{3}$ L. G. Tkachev ${ }^{4,9}$ R. Togoo ${ }^{16}$ M. Tluczykont ${ }^{10}$ N. Ushakov $^{12}$ A. Vaidyanathan ${ }^{7}$ P. A. Volchugov ${ }^{1}$ N. V. Volkov ${ }^{11}$ D. Voronin ${ }^{12}$ R. Wischnewski ${ }^{5}$ A. V. Zagorodnikov ${ }^{3}$ D. P. Zhurov ${ }^{3,17}$ I. I. Yashin ${ }^{2}$

${ }^{1}$ Skobeltsyn Institute of Nuclear Physics, Moscow State University, Moscow, 119991 Russia

${ }^{2}$ National Research Nuclear University MEPhI, Moscow, 115409 Russia

${ }^{3}$ Research Institute of Applied Physics, Irkutsk State University, Irkutsk, 664003 Russia

${ }^{4}$ Joint Institute for Nuclear Research, Dubna, Moscow oblast, 141980 Russia

${ }^{5}$ Deutsches Elektronen-Synchrotron DESY, Zeuthen, 15738 Germany

${ }^{6}$ Physics Department of the University of Torino and Istituto Nazionale di Fisica Nucleare, Torino, 10125 Italy

${ }^{7}$ Novosibirsk State University, Novosibirsk, 630090 Russia

${ }^{8}$ Budker Institute of Nuclear Physics, Siberian Branch, Russian Academy of Sciences, Novosibirsk, 630090 Russia

${ }^{9}$ Dubna University, Dubna, Moscow oblast, 141980 Russia

${ }^{10}$ Institut für Experimentalphysik, Universität Hamburg, Hamburg, D-22 761 Germany

${ }^{11}$ Altai State University, Barnaul, Altai krai, 656049 Russia

${ }^{12}$ Institute for Nuclear Research, Russian Academy of Sciences, Moscow, 117312 Russia

${ }^{13}$ Max Planck Institute for Physics, Munich, 80805 Germany

${ }^{14}$ Space Science Institute, Magurele, 077125 Romania

${ }^{15}$ Pushkov Institute of Terrestrial Magnetism, Ionosphere and Radio Wave Propagation, Russian Academy of Sciences, Troitsk, Moscow, 142190 Russia

${ }^{16}$ Institute of Physics and Technology Mongolian Academy of Sciences, Ulaanbaatar, Mongolia

${ }^{17}$ Irkutsk National Research Technical University, Irkutsk, Russia 